Proc. 19th Winter Workshop on

Nuclear Dynamics (2003) 000-000

\begin{tabular}{l}
\hline \hline 19th Winter Workshop \\
on Nuclear Dynamics \\
Breckenridge, Colorado, USA \\
February 8-15, 2003 \\
\hline \hline
\end{tabular}

\title{
Equilibrium in Heavy Ion Collisions
}

\author{
V. Koch and A. Majumder \\ Nuclear Science Division \\ Lawrence Berkeley National Laboratory \\ Berkeley, CA 94720, USA
}

\begin{abstract}
We discuss the question of equilibriation in heavy ion collisions and how it can be addressed in experiment

Keywords: Heavy Ion Collision, Equilibrium, Correlations

PACS: 25.75.-q, 24.10.Pa, 24.60.-k
\end{abstract}

\section{Introduction}

The purpose of relativistic heavy ion collisions is to produce and study new forms of matter. Therefore, one of the most basic requirements is that a system close to thermal equilibrium is generated in these collisions. Considerable effort has gone into this question with an emphasis on the description of particle abundances. Indeed, the hypothesis of a thermalized system (thermal model) is very successful in reproducing and predicting measured particle ratios of a wide range of energies and system sizes (for a recent review see Ref. [1]).

However, particle abundances provide only very limited information about the system and the actual (non-thermal) dynamics may simply be averaged out in these observables. This is the underlying idea of the statistical model by Fermi [2], which assumes that phase space dominates simple (single particle) observables of a multi-particle system. Indeed, the statistical approach not only works for heavy ion systems but also for $e^{+} e^{-}$or proton-proton collisions. In the latter cases, there is hardly any matter produced which is in equilibrium. Therefore, the success of the thermal model in reproducing the particle ratios is a necessary, but not sufficient, condition for the existence of matter close to equilibrium.

We could for instance imagine that a heavy ion reaction is simply a collection of many independent nucleon-nucleon collisions without any re-interaction of the secondary particles (Fig. 1(a)). This system would still look thermal in the particle ratios, but hardly constitute matter (for a more detailed discussion see Ref. [3]). The other extreme, depicted in Fig. 1(b), is matter equilibrated over the entire 


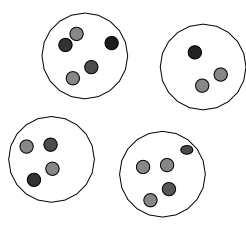

(a)

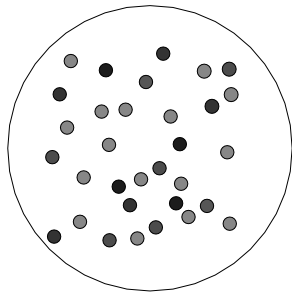

(b)

Fig. 1. Individual nucleon-nucleon collisions (a) and nucleus-nucleus collision (b).

volume of the system. Particle ratios alone cannot distinguish between these two scenarios. But are there additional measurements, such as multi-particle correlations, that allow us to distinguish between the two scenarios?

In the limit that each small system of Fig. 1(a) can be described in the grandcanonical approximation, there is no way to distinguish between the two scenarios by simply measuring final state particle yields and correlations. Even if the subsystems are separated by arbitrary distances. In this limit, the free energy of the system is just the sum of the free energies of any subdivision, i.e. correlations are absent. This is for instance the case for pions at high energies.

However, additional conserved charges, such as strangeness introduce correlations. In the scenario of Fig प(a), strangeness needs to be conserved in each small volume separately, thus reducing the yield of multiply strange hadrons, such as the Omega baryon [4]. This is illustrated in Fig 2] where we show the abundance of strange particles (normalized to the pion number). Up to a volume of about 20 times that of a nucleon-nucleon system, explicit strangeness conservation reduces the yield; most dramatically for the $S=-3$ states.

The question is then: can we utilize the correlations introduced by the strangeness conservation to determine the region over which strangeness is equilibrated? To address this issue let us discuss the following model. Consider a system of total volume $V_{f}$ divided into $p$ domains in which strangeness is explicitly conserved. Suppose further that each of these subsystems has the same temperature. This assumption is somewhat justified since it is expected that kinetic equilibrium is reached faster than chemical equilibrium. More importantly, in very high energy collisions of all species of 'in states' (e.g., $e^{+} e^{-}, p, p$ and $A, A$ ), the freeze-out temperature assumes an almost universal value of $170 \mathrm{MeV}$. Hence, it is reasonable to assume that each domain freezes out independently with a temperature $T \simeq 170$. Furthermore, let us denote by $V_{p}=V_{f} / p$ the volume of each domain. As we increase the number of domains or decrease the domain volume, the effect of local strangeness conservation becomes more prominent. As a result, the number of strange particles is reduced in 


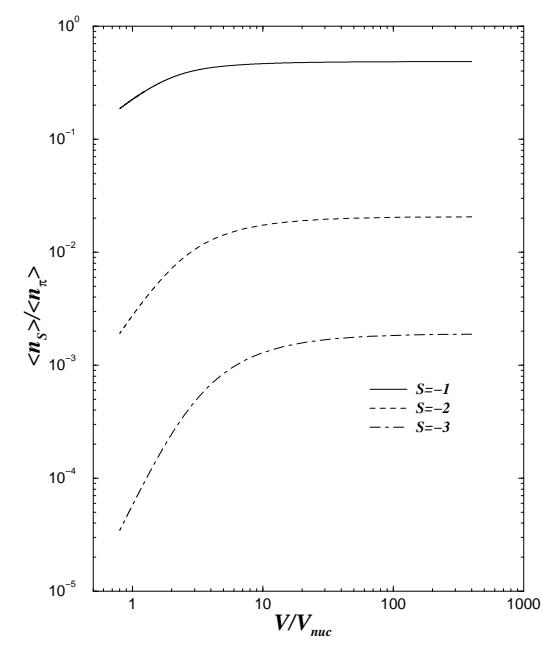

Fig. 2. Abundance of strange particles as a function of the volume.

the same fashion as depicted in Fig 2] (see also [4]). However, for domain volumes

$$
V_{p}>20 V_{n u c},
$$

the effect of strangeness conservation on the average particle number is small. Here, $V_{\text {nuc }}$ denotes the volume of a nucleon-nucleon collision. This is probably the case at RHIC, where hardly any centrality dependence of the strange particle yields is observed. At SPS energies the situation is different. There, NA57 [6] measures a strong centrality dependence of the $\Omega$-baryon yield, which is compatible with the rise of the $S=-3$ curve of Fig 2 (a) thus indicating a rather small strangeness equilibration volume $V \leq 20 V_{\text {nuc. }}$. even for the most central collisions.

So what can be done, if, as it is most likely the case at RHIC, the domain volume is larger than $20 V_{n u c}$ and the sensitivity of the single particle yield to the domain size becomes rather weak. The obvious idea is to measure many particle coincidences. If the domain is sufficiently large, such that, the production of three units of strangeness (such as an $\Omega$-baryon) is not affected by local strangeness conservation, then perhaps the production of 6 or 9 etc., units of strangeness is. This is demonstrated in Fig. 3] where we plot the probability distribution of $\Omega$ baryons for different number of domains. Obviously, for $N_{\Omega} \geq 5$, the sensitivity on the domain size is quite substantial. The probabilities to be measured are $P>10^{-6}$ and are thus, in principle, accessible in a heavy ion experiment, where of the order of $10^{6}$ events are being collected.

In Fig 3(b) we show the ratio of the $\Omega$ probability distribution over that of a Poisson distribution. The Poisson distribution is fixed by the average value of the 

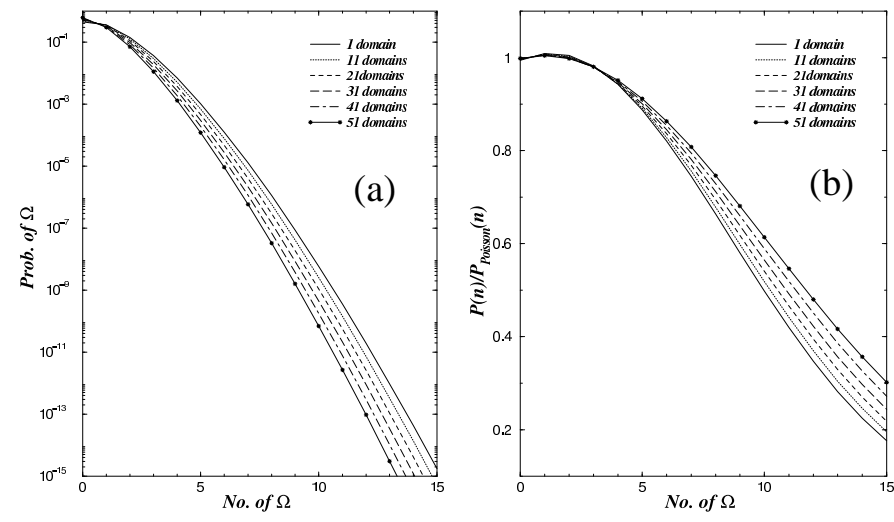

Fig. 3. Probability distribution for $\Omega$-baryon for different numbers of domains (a). The right panel (b) shows the deviation from a Poisson distribution.

true distribution. We find that for $N_{\Omega} \leq 5$ the probabilities are essentially given by the Poisson distribution. Actually, the more domains we have, the closer we are to a Poisson distribution. At first sight, this is entirely counter intuitive. Many domains means small domain volume which in turn implies that the explicit strangeness conservation should be most relevant. Thus the system should deviate maximally from a grand-canonical description. At the same time we find a Poissonian behavior, which is often considered an indication for a system in the grand-canonical limit.

The reason, we see Poisson like behavior in our case, is that for each small domain the probability to find an $\Omega$ is essentially binomial; either we find none or we find one.

$$
\begin{aligned}
P_{\text {domain }}(0) & =1-\epsilon \\
P_{\text {domain }}(1) & =\epsilon \\
P_{\text {domain }}(N>1) & \simeq 0
\end{aligned}
$$

The probability to find more than one can be ignored. And having many domains, this binomial distribution turns in to a Poisson distribution as long as $N_{\Omega} \leq N_{\text {domains. }}$. Furthermore, since the probability distribution falls off very rapidly (see Fig [3(a)), two particle observables are determined by exactly the region where the distribution is very close to Poisson. As an amusing consequence,

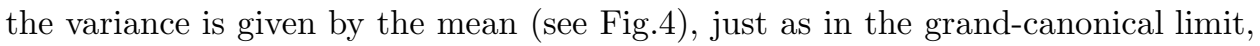
although we are in a region where explicit strangeness conservation is essential. Therefore, two particle observables do not provide new information about the size of the underlying domains. Quite to the contrary, they may lead to the wrong conclusion about the properties of the system at hand. 


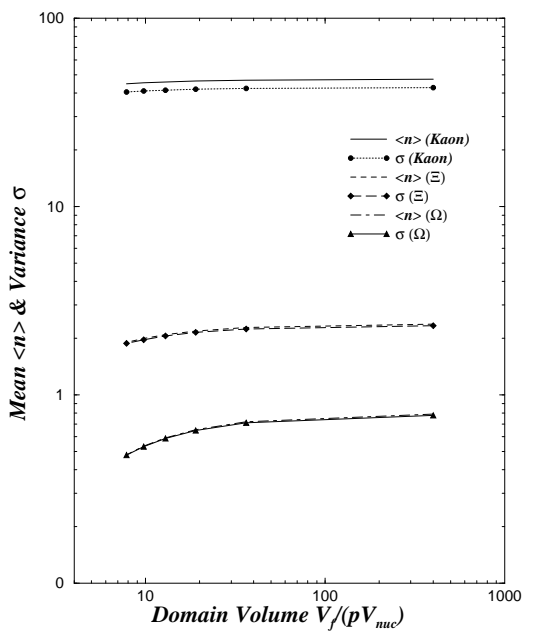

Fig. 4. Mean and variance for different strange particles as a function of domain size.

\section{Conclusions}

We have discussed the production of strange particles as a possible measure of the strangeness equilibrium volume. This volume will provide a lower bound on the equilibrium volume created in a heavy ion collision. We have shown, that the measurement of multiple $\Omega$-baryon final states with $N_{\Omega} \simeq 5$ can determine the size of this volume. We have further pointed out that two particle correlation will not provide new information compared to single particle measurements. In conclusion, to establish the degree of equilibrium reached in these collisions requires a very precise and dedicated measurement of multi-particle final states. To which extent this can be achieved in present heavy ion experiments remains to be seen.

\section{Acknowledgments}

This work was supported in part by the Natural Sciences and Engineering Research Council of Canada and in part by the Director, Office of Science, Office of High Energy and Nuclear Physics, Division of Nuclear Physics, and by the Office of Basic Energy Sciences, Division of Nuclear Sciences, of the U.S. Department of Energy under Contract No. DE-AC03-76SF00098. 


\section{References}

1. P. Braun-Munzinger et al., nucl-th/0304013

2. E. Fermi, Progr. Theoret. Phys. 5 (1950) 570.

3. V. Koch, Nucl. Phys. A A715 (2003) 108c.

4. S. Hamieh, K. Redlich and A. Tounsi, Phys. Lett. B486 (2000) 61

5. A. Majumder and V. Koch, nucl-th/0305047, subm. to PRC.

6. V. Manzari for the NA57 collaboration, these proceedings. 